\title{
Myristoylated Alanine-Rich C Kinase Substrate Accelerates TNF- $\alpha$-Induced Apoptosis in SH-SY5Y Cells in a Caspases-6 and/or -7-Dependent Manner
}

\author{
Atsuhiro Tanabe1,2*, Mitsuya Shiraishi' ${ }^{3}$, Yasuharu Sasaki² \\ ${ }^{1}$ Laboratory of Biochemistry, Department of Bioscience and Engineering, Shibaura Institute of Technology, \\ Saitama, Japan \\ ${ }^{2}$ Laboratory of Pharmacology, School of Pharmacy, Kitasato University, Tokyo, Japan \\ ${ }^{3}$ Department of Veterinary Pharmacology, Faculty of Agriculture, Kagoshima University, Kagoshima, Japan \\ Email: ${ }^{*}$ tanabea@shibaura-it.ac.jp
}

Received 20 July 2015; accepted 21 August 2015; published 24 August 2015

Copyright (C) 2015 by authors and Scientific Research Publishing Inc.

This work is licensed under the Creative Commons Attribution International License (CC BY). http://creativecommons.org/licenses/by/4.0/

(c) (i) Open Access

\section{Abstract}

Cell proliferation, differentiation, and the elimination of unnecessary cells by apoptosis occur in the development of the nervous system. It is reported that brain dysplasia appears as the results of myristoylated alanine-rich C kinase substrate (MARCKS) knockout or the mutant mouse. We therefore expect that MARCKS participates in the development of the nervous system. However, the mechanism underlying such participation has not been identified. In this study, we observed the effects of the overexpression of MARCKS or unphosphorylatable MARCKS on cell proliferation and TNF- $\alpha$-induced apoptosis in neuroblastoma SH-SY5Y cells. Furthermore, we restrained MARCKS expression by the RNAi method. In the results, MARCKS-overexpressing cells and not unphosphorylatable MARCKS-overexpressing cells showed increased cell proliferation rates. On the other hand, the RNAi decreased the proliferation of MARCKS-knocked down SH-SY5Y cells. These results indicated that MARCKS-overexpressing cells were more sensitive to TNF- $\alpha$ than normal SH-SY5Y cells. Moreover, in MARCKS-overexpressing cells TNF- $\alpha$-induced apoptosis was inhibited by caspase- 6 and -7 inhibitors but not by caspase- 3 inhibitor. These results suggested that MARCKS participated in TNF- $\alpha$-induced apoptosis in a caspase- 6 and/or -7-dependent manner.

\section{Keywords}

Apoptosis, MARCKS, Caspase, Phosphorylation

\footnotetext{
${ }^{*}$ Corresponding author.
}

How to cite this paper: Tanabe, A., Shiraishi, M. and Sasaki, Y. (2015) Myristoylated Alanine-Rich C Kinase Substrate Accelerates TNF- $\alpha$-Induced Apoptosis in SH-SY5Y Cells in a Caspases-6 and/or -7-Dependent Manner. Advances in Bioscience and Biotechnology, 6, 572-582. http://dx.doi.org/10.4236/abb.2015.68060 


\section{Introduction}

Myristoylated alanine-rich C kinase substrate (MARCKS), which has a phosphorylated site domain (PSD), is cloned as a protein kinase C (PKC) substrate. It is known that several kinases, including ROCK, PKA, and MAPK, phosphorylate MARCKS in vitro [1]. Furthermore, we showed that PKC phosphorylated MARCKS not only directly but also through the RhoA/ROCK pathway in SH-SY5Y neuroblastoma cells [2].

MARCKS seems to be implicated in cell motility [3], secretion [4], membrane trafficking [5], and mitogenesis [6] through the regulation of cytoskeletal structure. Unphosphorylated MARCKS binds to actin directly and crosslinks F-actin, whereas phosphorylated MARCKS loses actin binding and polymerization activities [7]. Moreover, it is reported that MARCKS releases phosphatidylinositol-4,5-bisphosphate (PIP $\left.{ }_{2}\right)$ through PSD in a phosphorylation-dependent manner [8] by which actin dynamics is regulated. Nevertheless, the exact mechanisms underlying the physiological roles of MARCKS are still unclear.

Stumpo and his colleagues reported that homozygous deletions of the MARCKS gene in mice led to abnormal brain development and perinatal death [9]. That report indicated that MARCKS played a pivotal role in the normal development processes of neurulation, hemisphere fusion, forebrain commissure formation, and the formation of cortical and retinal laminations. During nervous system development, neuronal cells not only proliferate but undergo a process of apoptosis [10]. We predicted that MARCKS was associated with apoptosis in the developing brain.

A lot of physiological phenomena are associated with apoptosis (e.g., germ cell development, elimination of tumor cells and DNA-damaged cells, and blood cell exchange). In these physiological phenomena, several apoptotic cascades are partially known. Tumor necrosis factor (TNF)- $\alpha$ and Fas ligand are examples of apoptosis-inducing factors [11]. These factors bind to specific receptors (so-called death receptors) on the cell surface, by which signals are transmitted into the cells. Although different factors are activated depending on the cause of the apoptosis, it is well known that caspases are the principal proteins in apoptosis.

In this report, we show that MARCKS is associated with both basal apoptotic levels and TNF- $\alpha$-induced apoptosis in neuroblastoma cells. Moreover, we report that MARKCS-related apoptosis is independent from caspase-3 but dependent on caspase-6 and/or -7 .

\section{Materials and Methods}

\subsection{Cell Culture and Proliferation Assays}

SH-SY5Y human neuroblastoma cells (nor-cells), GFP-expressing SH-SY5Y cells (GFP-OE-cells), GFP-fused wild-type MARCKS-overexpressing SH-SY5Y cells (wtMAR-OE-cells), and GFP-fused unphosphorylatable MARCKS-overexpressing cells (m3MAR-OE-cells) [12] were maintained in Dulbecco's modified Eagle's medium supplemented with $10 \%$ fetal bovine serum, 100 units $/ \mathrm{ml}$ penicillin, and $100 \mu \mathrm{g} / \mathrm{ml}$ streptomycin at $37^{\circ} \mathrm{C}$ in a humidified atmosphere with $5 \% \mathrm{CO}_{2}$. Proliferation assays were performed by comparing the cell numbers between 2 and 5 days after the cells were plated in complete medium. All cell lines were plated on 24-well plates at a density of $5 \times 10^{3} /$ well. The cells in each well (from 3 wells per cell line) were counted.

\subsection{RNA Interference}

MARCKS-specific double-stranded RNA oligonucleotides, each consisting of a 25-nucleotide sense sequence and a 25-nucleotide antisense sequence, were purchased from Invitrogen. The sequences for MARCKS siRNA were as follows: sense, 5'-uucgcugcggucuuggagaacuggg-3'; antisense, 5'-cccaguucuccaagaccgcagcgaa-3'.

SH-SY5Y cells were plated on 24-well plates at a density of $5 \times 10^{3}$ cells/well. After $24 \mathrm{~h}, 6.0 \mathrm{pmol}$ of the MARCKS-specific or negative control siRNA (Invitrogen) was transfected to the cells using Lipofectamine2000 (Invitrogen).

\subsection{Western Blotting Analysis}

Trichloroacetic acid precipitants were subjected to Western blotting analyses as previously described [2]. The phosphorylation of MARCKS was detected with pS159-Mar-Ab [1] (1:5000 with Can Get Signal solution 1 (Toyobo)). Signals were detected by Chemi Lumi One (Nacalai Tesque) and Light Capture (ATTO). Densitometric analyses were performed using CS Analyzer (ATTO) software. 


\subsection{Apoptosis Detection}

The cells were seeded on cover glasses in $35 \mathrm{~mm}$ dishes at a density of $1.0 \times 10^{5}$ cells/dish. After 2 days, the medium was changed to FBS-free DMEM with $1 \mu \mathrm{M}$ TNF- $\alpha$ and incubated for $24 \mathrm{~h}$. The cells were washed with ice-cold PBS (-) and fixed with 4\% paraformaldehyde/4 mM EGTA/4\% sucrose for $1 \mathrm{~h}$ at room temperature. The apoptotic cells were stained by the Apop Tag Red In Situ Apoptosis Detection Kit (Chemicon International), and the cover glasses were mounted on slide glasses with DAPI-containing Vectashield (Vector Laboratories) and a Biozero8000 fluorescence microscope (Keyence). The apoptotic cells were counted and the results were expressed as means \pm SEM of the mean. The statistical differences for the response against TNF- $\alpha$ treatment in each cell line were determined by the two-sided Student's t-test. The data were analyzed using the Tukey-Kramer method for all pairwise comparisons between the TNF- $\alpha$ treatment mean. A difference of $p<0.05$ was considered significant.

\section{Results}

\subsection{MRACKS Expression's Effects on the Cell Proliferation Rate}

Because it has been reported that MARCKS is associated with the proliferation of several cell types [13]-[15], we compared the growth speed among normal SH-SY5Y cells (nor-cells), GFP-expressing cells (GFP-OE-cells), GFP-fused wild-type MARCKS-overexpressing cells (wtMAR-OE-cells), and GFP-fused unphosphorylatable MARCKS (m3MARCKS)-overexpressing cells (m3MAR-OE-cells) [12]. We counted the cells at 2 days and 5 days after seeding.

Figure 1(a) shows the cell numbers at 5 days after seeding relative to those at 2 days after seeding. According to these results, the cell doubling times were calculated (nor-cell, $1.45 \pm 1.21$; GFP-OE-cell, $1.50 \pm 0.20$; wtMAR-OE-cell, $1.09 \pm 0.31$; m3MAR-OE-cell, $1.47 \pm 0.17$ days). The results indicated that MARCKS overexpression and its phosphorylation accelerate the growth rate of SH-SY5Y cells.

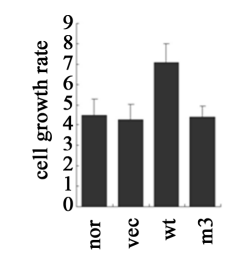

(a)

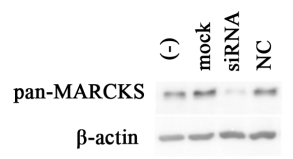

(b)

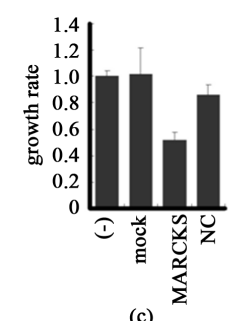

Figure 1. MARCKS involved in the growth of SH-SY5Y cells. (a) All cell lines (nor-, GFP-OE-, wtMAR-OE-, and m3MAR-OE-cells) were plated on 24-well plates at a density of $5 \times 10^{3} /$ well. The cells per well at 2 and 5 days later were counted $(\mathrm{n}=3)$. The cells at 5 days after were divided by those at 2 days after, and the growth rate was expressed as the mean \pm SD. ${ }^{*} p<0.05$ compared with nor-cells; (b) SH-SY5Y cells were transfected with only transfection reagent (mock), MARCKS-specific siRNA (MARCKS), or negative control siRNA (NC). After $24 \mathrm{~h}$, Western blotting assays were performed; (c) The MARCKS levels of (b) were quantified by densitometric analyses. The data for the nontreated cells were taken as 1; data represent mean \pm SEM of four experiments. ${ }^{* *} p<0.01$ compared with nontreated cells (nor: nor-cell; vec: GFP-OE-cell; wt: wtMAR-OE-cell; m3: m3MAR-OE-cells). 
Next we observed the effects of MARCKS knockdown on cell growth (Figure 1(b)). At first we observed the effects of three MARCKS siRNA from Stealth RNAi (Invitrogen) and most effective sequence, that sequence is shown in Materials and Methods, is decided to use mainly hereafter (data not shown).

The growth of MARCKS-knocked down cells was slower than that of control cells (Figure 1(c)) and other siRNAs showed same but little slight effects. However, the cell-cycle phases did not differ among these four kinds of cells in flow cytometry (data not shown). These results showed that MARCKS is involved in the proliferation of SH-SY5Y cells.

\subsection{MARCKS Is Involved in TNF- $\alpha$-Induced Apoptosis of SH-SY5Y Cells}

It is well recognized that some proteins are involved in both apoptosis and cell-cycle regulation [16]-[18]. Therefore, we observed the effects of MARCKS on apoptosis. In this report we used TNF- $\alpha$, which is an important mediator of inflammation, apoptosis, and the development of secondary lymphoid structures, to induce apoptosis. The immunocytochemistry showed that, following $24 \mathrm{~h} \mathrm{TNF}-\alpha$ treatment, more than $60 \%$ of cells expanded and that the apoptotic cells were condensed (Figure 2(a) arrowheads). Next we investigated the difference in the apoptosis rate among these four cell types when we treated these cells with TNF- $\alpha$. As we expected, TNF- $\alpha$ induced apoptosis in all of the kinds of cells we used. Especially in wtMAR-OE-cells, apoptosis was induced remarkably. On the other hand, the apoptosis rate of m3MAR-OE-cells was similar to those of norand GFP-OE-cells (Figure 2(b) and Figure 2(c)).
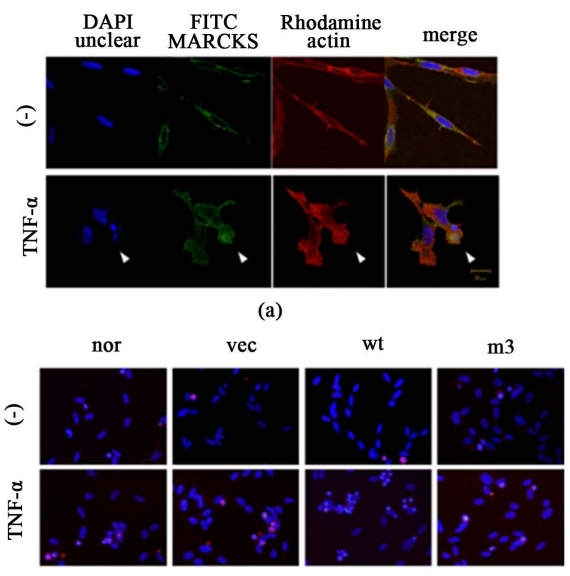

(b)

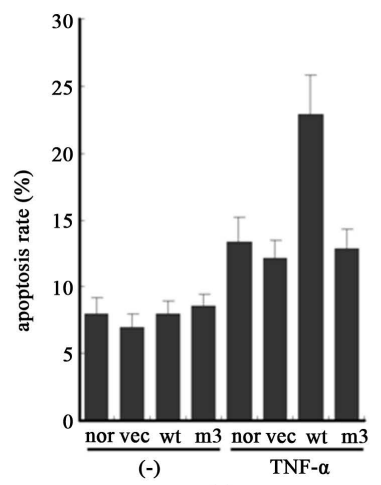

(c)

Figure 2. Phosphorylatable MARCKS accelerates TNF- $\alpha$-induced apoptosis. (a) The nor-cells were exposed to TNF- $\alpha$ for $24 \mathrm{~h}$. The nuclei, MARCKS, and actin were detected with DAPI, MARCKS-specific antibody and FITC-conjugated secondary antibody, and rhodamine-conjugated phalloidin, respectively. The arrow indicates apoptotic cell; (b) All cell lines (nor-, GFP-OE-, wtMAR-OE-, and m3MAR-OE-cells) were exposed to TNF- $\alpha$ for $24 \mathrm{~h}$. The apoptotic cells were stained by the ApopTag Red In Situ Apoptosis Detection Kit (red) and the nuclei were stained with DAPI (blue); (c) The apoptotic cells in each cell line in B were counted, and the rates are shown. The data represent the mean \pm SD of four experiments. ${ }^{*} p<0.05$ compared with TNF- $\alpha$-treated nor-cells. 
Next we knocked down MARCKS in SH-SY5Y cells with MARCKS-specific siRNA and treated the cells with TNF- $\alpha$. The immunocytochemistry shows that MARCKS expression is repressed with MARCKS-specific siRNA but not with negative control siRNA (NC) (Figure 3(a)). The MARCKS knockdown increased the basal apoptosis rate while NC showed no effect. On the other hands, in the case of TNF- $\alpha$-induced apoptosis MARCKS siRNA did not show significant differences with both mock and NC (Figure 3(b) left panel). As we thought in these sensitive conditions such adding damages to the cells by transfection regent and apoptosis-inducing regent even NC affects apoptosis rate, we calculated relative effects as fold (Figure 3(b) right panel). It shows that MARKCS siRNA almost completely inhibits TNF- $\alpha$-induced apoptosis. These results strongly indicate that MARCKS is involved in apoptosis.

\subsection{Phosphorylated MARCKS Is Involved in TNF- $\alpha$-Induced Apoptosis}

The outcome shown in Figure 2 indicates that wild-type MARCKS accelerates TNF- $\alpha$-induced apoptosis but m3MARCKS does not. Thus we thought that phosphorylated MARCKS is involved in the TNF- $\alpha$-induced apoptosis cascade. To confirm our opinion, we first ascertained the pan-MARCKS expression levels in these cells. In nor- and GFP-OE-cells, the endogenous MARCKS (endo-MARCKS) amount increased slightly, although those of wtMAR-OE- and m3MAR-OE-cells did not change. On the other hand, the GFP-fused MARCKS (exo-MARCKS) amount in both wtMAR-OE- and m3MAR-OE-cells increased after TNF- $\alpha$ treatment (Figure 4(a) upper panel). Nonetheless, the amount of exo-MARCKS was smaller than that of endoMARCKS. We also observed the phosphorylation level of MARCKS in TNF- $\alpha$-treated cells. Without stimulation, exo-MARCKS in wtMAR-OE-cells was phosphorylated. In the early phase after TNF- $\alpha$ stimulation $(0-1 \mathrm{~h})$,

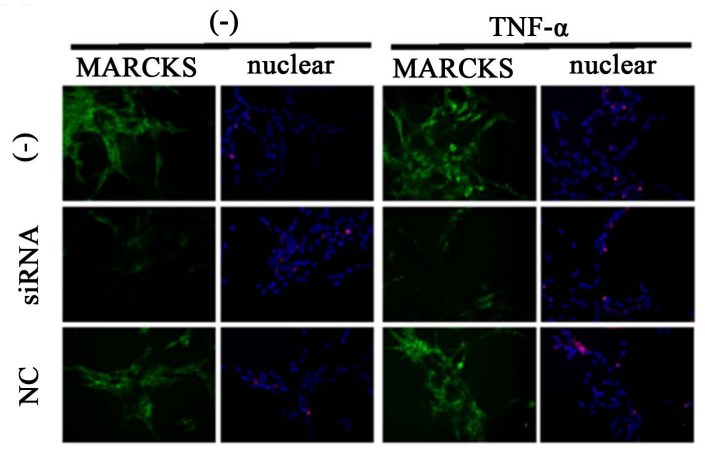

(a)
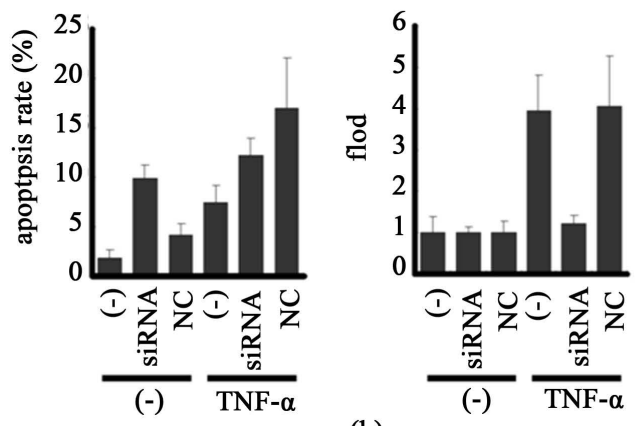

(b)

Figure 3. MARCKS knockdown reduces TNF- $\alpha$-induced apoptosis. (a) MARCKS in the nor-cells was knocked down with MARCKS-specific siRNA. The cells were exposed to TNF- $\alpha$ for $24 \mathrm{~h}$. The nuclei and MARCKS were detected with DAPI and with MARCKS-specific antibody and FITC-conjugated secondary antibody, respectively. The apoptotic cells were stained with the ApopTag Red In Situ Apoptosis Detection Kit; (b) left panel. The apoptotic cells in A were counted and the rates are shown. The data represent the mean \pm SEM of three to six experiments. ${ }^{*} p<0.05$ compared with TNF- $\alpha$-treated nor-cells (nor: nor-cell; vec: GFP-OE-cell; wt: wtMAR-OE-cell; m3: m3MAR-OE-cells). 


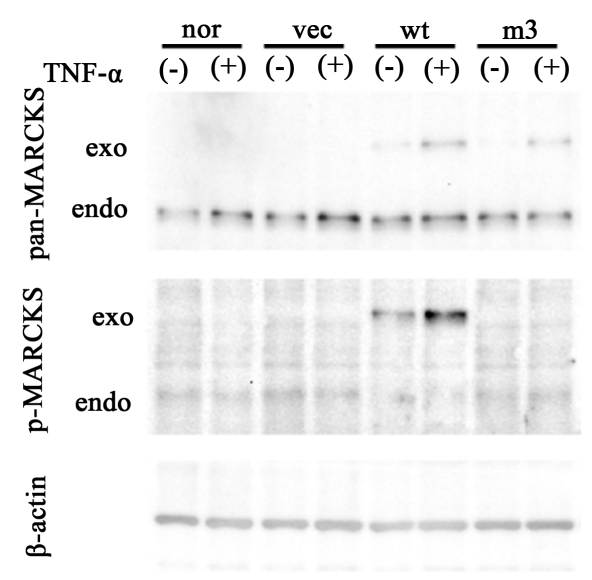

(a)

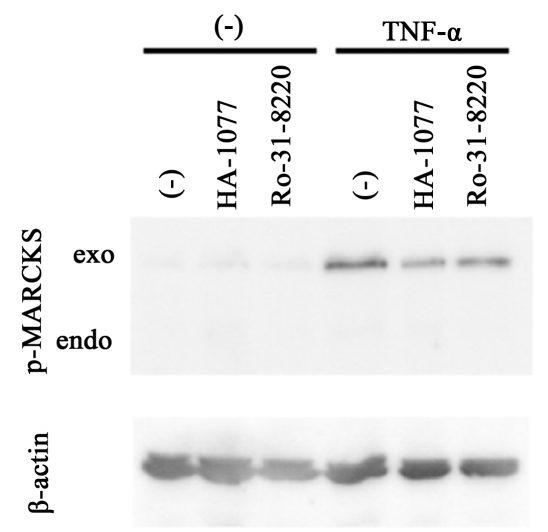

(b)

Figure 4. TNF- $\alpha$ induces MARCKS phosphorylation through ROCK and PKC. (a) All cell lines (nor-, GFP-OE-, wtMAROE-, and m3MAR-OE-cells) were exposed to TNF- $\alpha$ for $24 \mathrm{~h}$. MARCKS, phosphorylated MARCKS, and $\beta$-actin were detected by Western blotting analyses using anti-MARCKS antibody, pS159-Mar-Ab, and anti- $\alpha$-actin antibody, respectively; (b) wtMAR-OE-cells, pretreated with ROCK inhibitor HA-1077 or PKC inhibitor Ro-31-8220, were exposed to TNF- $\alpha$ for 24 h. Phosphorylated MARCKS and $\alpha$-actin were detected by Western blot analyses using pS159-Mar-Ab and anti- $\alpha$-actin antibody, respectively (nor: nor-cell; vec: GFP-OE-cell; wt: wtMAR-OE-cell; m3: m3MAR-OE-cells).

no increase in MARCKS phosphorylation was detected (data not shown). Nonetheless, $24 \mathrm{~h}$ after TNF- $\alpha$ treatment, only exo-MARCKS of wtMAR-OE-cells was phosphorylated (Figure 4(a), middle panel). No change in the $\beta$-actin amount with TNF- $\alpha$ treatment was detected (Figure 4(a), lower panel). The TNF- $\alpha$-induced phosphorylation of exo-MARCKS in wtMAR-OE-cells was slightly reduced by pretreatment with either ROCK inhibitor HA-1077 or PKC inhibitor Ro-31-8220 (Figure 4(b)).

\subsection{In wtMAR-OE-Cells, Caspase- 6 and/or -7, but Not Caspase-3, Is Involved in TNF- $\alpha$-Induced Apoptosis}

Although the apoptosis cascade is not perfectly understood, caspase- 3 is considered one of the most important proteins in apoptosis. Therefore, at first we used caspase- 3 inhibitor to observe the participation of caspase- 3 in the apoptosis of TNF- $\alpha$-treated cells. In nor-, GFP-OE-, and m3MAR-OE-cells, TNF- $\alpha$-induced apoptosis was decreased with a caspase-3 inhibitor z-DQMD-fmk. In wtMAR-OE-cells, on the other hand, TNF- $\alpha$-induced apoptosis was not affected by z-DQMD-fmk (Figure 5).

Because caspases- 6 and -7 are classified as effector caspases, as is caspase-3, we investigated the possibility that caspase- 6 or -7 is involved in TNF- $\alpha$-induced apoptosis in wtMAR-OE-cells. Before TNF- $\alpha$ treatment, we treated the cells with z-VEID-fmk or z-VEVD-fmk, which are caspase-6 and caspase-3/7 inhibitors, respectively. Both z-VEID-fmk and z-VEVD-fmk reduced the apoptosis rate in wtMAR-OE-cells (Figure 6). Another 


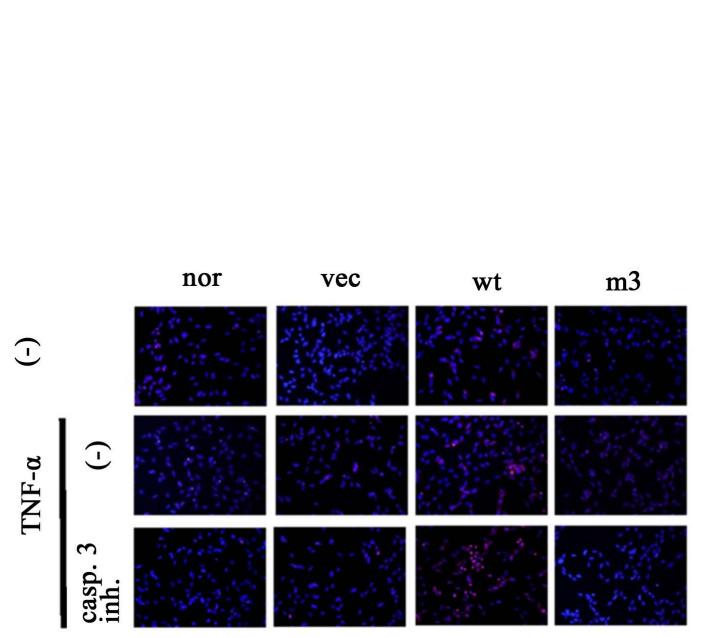

(a)

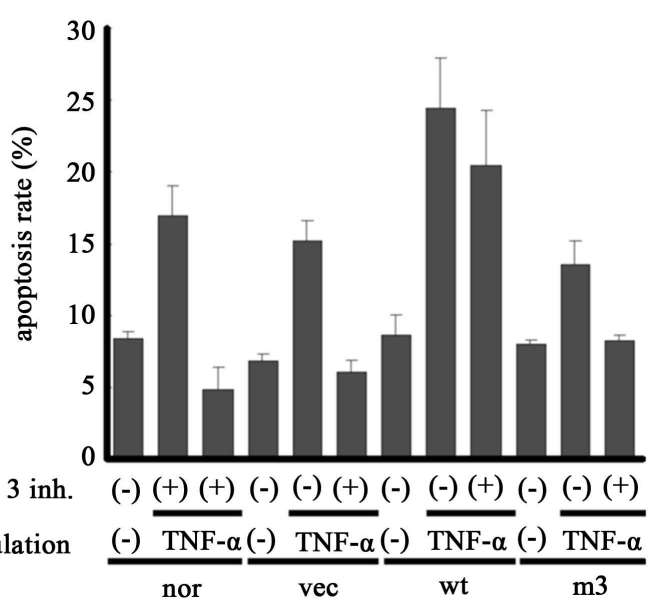

(b)

Figure 5. MARCKS upregulates the TNF- $\alpha$ induced apoptosis of caspase-3 independently. nor-cells pretreated with or without caspase-3 inhibitor z-DQMD-fmk were exposed to TNF- $\alpha$ for $24 \mathrm{~h}$. The apoptotic cells were stained by the ApopTag Red In Situ Apoptosis Detection Kit (red), and the nuclei were stained with DAPI (blue); (b) The apoptotic cells in each cell line in A were counted, and the rate is shown. The data represent the mean \pm SEM of three experiments. ${ }^{* *} p<0.01$ and ${ }^{*} p<$ 0.05 compared with each TNF- $\alpha$-treated cell line without caspase-3 inhibitor treatment (nor: nor-cell; vec: GFP-OE-cell; wt: wtMAR-OE-cell; m3: m3MAR-OE-cells).

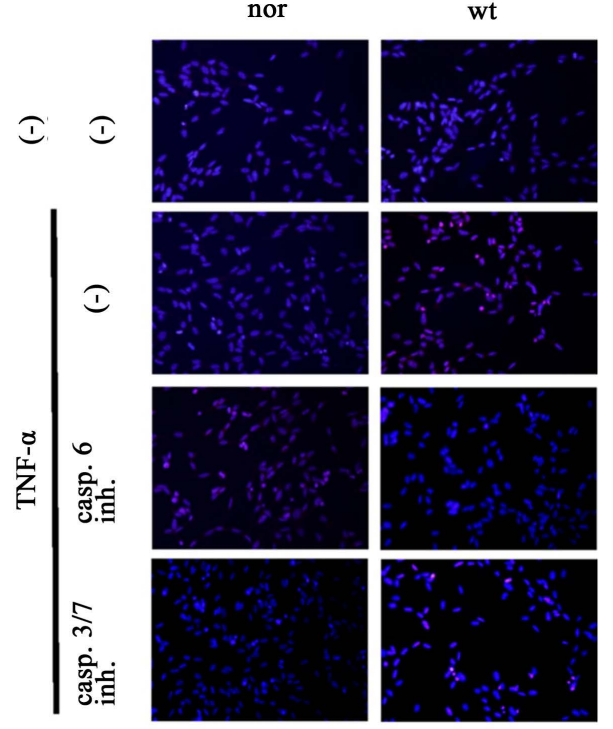

(a)

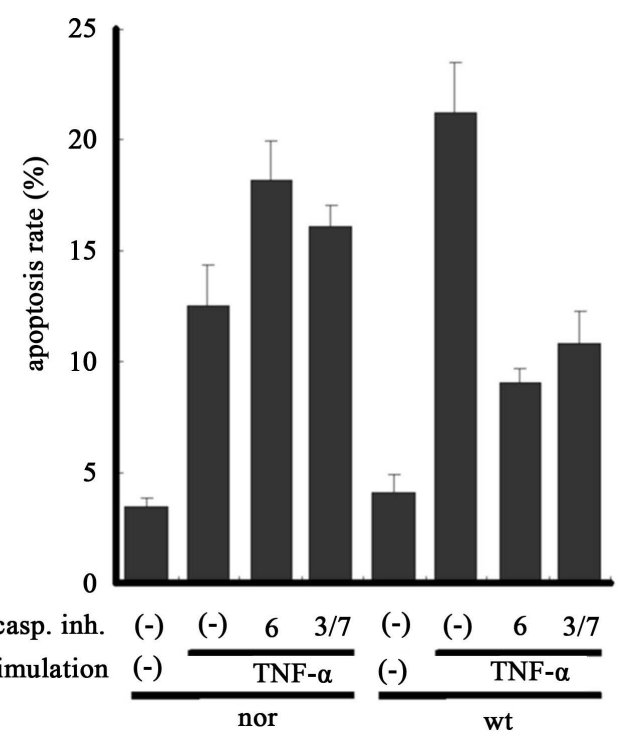

(b)

Figure 6. MARCKS upregulates TNF- $\alpha$-induced apoptosis of caspase- 6 and/or caspase-7 dependently. The nor-cells pretreated with or without caspase-3 inhibitor z-VEID-fmk or caspase-3/7 inhibitor z-VEVD-fmk were exposed to TNF- $\alpha$ for 24 h. The apoptotic cells were stained by the ApopTag Red In Situ Apoptosis Detection Kit (red), and the nuclei were stained with DAPI (blue); (b) The apoptotic cells in each cell line in A were counted, and the rate is shown. The data represent the mean \pm SEM of three experiments. ${ }^{* *} p<0.01$ compared with TNF- $\alpha$-treated wtMAR-OE-cell line without caspase-3 inhibitor treatment (nor: nor-cell; wt: wtMAR-OE-cell).

caspase-3/7 inhibitor 5-[(S)-(-)-2-(Methoxymethyl)pyrrolidino] sulfonylisatin also inhibited the induction of apoptosis by TNF- $\alpha$ in wtMAR-OE-cells (data not shown) as well as z-VEVD-fmk. These results suggest that caspases-6 and/or 7 are involved in the apoptosis cascade in TNF- $\alpha$-treated wtMAR-OE-cell.

Consequently, we consider that phosphorylated MARCKS is involved in TNF- $\alpha$-induced apoptosis through caspases-6 and/or -7 . 


\section{Discussion}

The importance of MARCKS in the developing brain has been reported, and recently it was revealed that MARCKS modulates radial progenitor placement and proliferation in the developing brain [14]. It is well known that MARCKS is associated with proliferation in several types of cells besides glial cells [19] [20]. However, the relationship between MARCKS and neuronal cell proliferation has not been clarified. In this study, in SH-SY5Y cells, overexpression of MARCKS increased the proliferation rate, while MARCKS knockdown reduced it. Moreover, unphosphorylatable MARCKS overexpressed cells did not change the growth rate. Our findings suggest that MARCKS is involved in the growth of not only glial cells but also neuronal cells.

In the developing brain apoptosis, as well as cell proliferation, is an unavoidable cell response. In this study, we showed that wild-type MARCKS-overexpressing cells (wtMAR-OE-cells) upregulate TNF- $\alpha$-induced apoptosis. It is well known that MARCKS is phosphorylated by PKC. Actually, TNF- $\alpha$-induced MARCKS phosphorylation was partially inhibited by PKC inhibitor Ro-31-8220. Therefore, we thought that PKC plays an important role in phosphorylated MARCKS-involved apoptosis. Several PKCs are involved in TNF- $\alpha$-induced apoptosis in several cell types [21]-[23]. In the future, an issue to examine is which PKC isoform is involved in TNF- $\alpha$-treated SH-SY5Y and how MARCKS is associated with it.

In this study we showed that ROCK inhibitor HA-1077 also inhibited TNF- $\alpha$-induced MARCKS phosphorylation at least partially. Besides PKC, ROCK is a candidate for kinase mediating MARCKS phosphorylation in the TNF- $\alpha$-induced apoptosis. Because Mong and his colleagues reported that TNF- $\alpha$ activates JNK through the Rho/ROCK pathway [24], it is reasonable to infer that MARCKS is phosphorylated downstream from TNF- $\alpha$. TNF- $\alpha$ also activates MAPK in several signal pathways [25]-[27] including the apoptotic cascade [28] [29]. It is reported that MAPK phosphorylates MARCKS in vitro [1] [30]. Moreover, Nwariaku and his colleagues indicated that ROCK inhibition attenuated TNF- $\alpha$-induced MAPK activation [31]. Thus, ROCK is closely related to TNF- $\alpha$. Previously we showed that PKC phosphorylates MARCKS through ROCK at least partially [2]. In the case of TNF- $\alpha$-induced apoptosis, PKC may activate ROCK and would consequently induce the phosphorylation of MARCKS.

MARCKS knockdown also increased apoptosis without TNF- $\alpha$ treatment. In the case of muscle cell spreading, which is mediated by integrin, MARCKS was localized soon after cell adhesion to the focal adhesion site and was phosphorylated by PKCs, leading to translocation from the membrane to cytosol [32]. Cell adhesion involving integrin was associated closely with TNF- $\alpha$-induced apoptosis [33]. It is possible that MARCKS regulates the basal apoptosis level through integrin-associated cell adhesion.

Talen et al. have reported that TNF- $\alpha$ induces the synthesis, myristoylation, and phosphorylation of MARCKS in neutrophils [34]. In our study, however, a slight increase in endo-MARCKS was recognized in nor- and GFP-OE-cells. On the other hand, although the exo-MARCKS amount was elevated by TNF- $\alpha$, endoMARCKS did not increase in wtMAR-OE- or m3MAR-OE-cells. We inserted MARCKS cDNA upstream from GFP, which is regulated under a cytomegalovirus (CMV) promoter in pEGFP-N1 to construct the GFPMARCKS expression plasmid, and the CMV promoter is activated by TNF- $\alpha$ [35]. Therefore, TNF- $\alpha$ stimulation upregulates exo-MARCKS expression. Taken together, the results indicate that TNF- $\alpha$ raised the total MARCKS amount slightly in all types of cells. Although TNF- $\alpha$ elevated the exo-MARCKS amount, the increase was very small compared with the endo-MARCKS amount. However, the phosphorylated exo-MARCKS amount was several times larger than the phosphorylated endo-MARCKS amount. We cannot explain why exo-MARCKS is phosphorylated more easily than endo-MARCKS. However, from a different viewpoint we might be able to create a highly phosphorylated MARCKS expression system.

Caspase activation is a critical event in the onset of apoptosis [36]. Fourteen caspases have been identified to date [37]. According to the roles in apoptosis, caspases are divided roughly into two groups: initiator caspases and effector caspases. Caspases-2, -8, $-9,-10$, and -12 are initiator caspases. The apoptotic signals through the initiator caspases converge on effector caspases (caspases-3, 6, and 7). Although the roles of these caspases are clearly different, their activation mechanisms are remarkably similar [38]. All caspases recognize specific four-residue sequences and cleave peptide bonds located strictly after an Asp group. Inactive procaspases are digested by initiator caspases and activated. These active effector caspases digest their own substrates, so-called death substrates. In this study, we showed that inhibition of caspase- 6 and -7 , but not -3 , reduced the TNF- $\alpha$ induced apoptosis of wtMAR-OE-cells. However, the reduction of MARCKS amount which means MARCKS digestion was not detected. It remains unclear how MARCKS affects apoptosis. 


\section{Conclusion}

Taken together, the present results show that MARCKS phosphorylation is involved in TNF- $\alpha$-induced neuronal cell apoptosis. To our knowledge, this is the first report to show the association of MARCKS with apoptosis. This novel apoptosis pathway will lead to the elucidation of apoptosis mechanisms in neurons and in brain development.

\section{Acknowledgements}

We thanks Dr. Naoaki Saito for wtMARCKS and m3MARCKS express plasmid.

\section{References}

[1] Nagumo, H., Ikenoya, M., Sakurada, K., Furuya, K., Ikuhara, T., Hiraoka, H. and Sasaki, Y. (2001) Rho-Associated Kinase Phosphorylates MARCKS in Human Neuronal Cells. Biochemical and Biophysical Research Communications, 280, 605-609. http://dx.doi.org/10.1006/bbrc.2000.4179

[2] Tanabe, A., Kamisuki, Y., Hidaka, H., Suzuki, M., Negishi, M. and Takuwa, Y. (2006) PKC Phosphorylates MARCKS Ser159 Not Only Directly but Also through RhoA/ROCK. Biochemical and Biophysical Research Communications, 345, 156-161. http://dx.doi.org/10.1016/j.bbrc.2006.04.082

[3] Zhao, Y., Neltner, B.S. and Davis, H.W. (2000) Role of MARCKS in Regulating Endothelial Cell Proliferation. American Journal of Physiology-Cell Physiology, 279, C1611-C1620.

[4] Blackshear, P.J. (1993) The MARCKS Family of Cellular Protein Kinase C Substrates. Journal of Biological Chemistry, 268, 1501-1504.

[5] McLaughlin, S. and Aderem, A. (1995) The Myristoyl-Electrostatic Switch: A Modulator of Reversible Protein-Membrane Interactions. Trends in Biochemical Sciences, 20, 272-276. http://dx.doi.org/10.1016/S0968-0004(00)89042-8

[6] Elzagallaai, A., Rose, S.D. and Trifaro, J.M. (2000) Platelet Secretion Induced by Phorbol Esters Stimulation Is Mediated through Phosphorylation of MARCKS: A MARCKS-Derived Peptide Blocks MARCKS Phosphorylation and Serotonin Release without Affecting Pleckstrin Phosphorylation. Blood, 95, 894-902.

[7] Hartwig, J.H., Thelen, M., Rosen, A., Janmey, P.A., Nairn, A.C. and Aderem, A. (1992) MARCKS Is an Actin Filament Crosslinking Protein Regulated by Protein Kinase C and Calcium-Calmodulin. Nature, 356, 618-622. http://dx.doi.org/10.1038/356618a0

[8] Wang, J., Arbuzova, A., Hangyás-Mihályné, G. and McLaughlin, S. (2001) The Effector Domain of Myristoylated Alanine-Rich C Kinase Substrate Binds Strongly to Phosphatidylinositol 4,5-Bisphosphate. Journal of Biological Chemistry, 276, 5012-5019. http://dx.doi.org/10.1074/jbc.M008355200

[9] Stumpo, D.J., Bock, C.B., Uttle, J.S. and Blackshear, P.J. (1995) MARCKS Deficiency in Mice Leads to Abnormal Brain Development and Perinatal Death. Proceedings of the National Academy of Sciences of the United States of America, 92, 944-948. http://dx.doi.org/10.1073/pnas.92.4.944

[10] Cowan, V.M., Fawcett, J.W., O’Leary, D.D.M. and Stanfield, B.B. (1984) Regressive Events in Neurogenesis. Science, 225, 1258-1265. http://dx.doi.org/10.1126/science.6474175

[11] Gruss, H.J. and Dower, S.K. (1995) Tumor Necrosis Factor Ligand Superfamily: Involvement in the Pathology of Malignant Lymphomas. Blood, 85, 3378-3404.

[12] Tanabe, A., Shiraishi, M., Negishi, M., Saito, N., Tanabe, M. and Sasaki, Y. (2012) MARCKS Dephosphorylation Is Involved in Bradykinin-Induced Neurite Outgrowth in Neuroblastoma SH-SY5Y Cells. Journal of Cellular Physiology, 227, 618-629. http://dx.doi.org/10.1002/jcp.22763

[13] McGill, C.J. and Brooks, G. (1997) Expression and Regulation of 80K/MARCKS, a Major Substrate of Protein Kinase C, in the Developing Rat Heart. Cardiovascular Research, 34, 368-376. http://dx.doi.org/10.1016/S0008-6363(97)00041-2

[14] Weimer, J.M., Yokota, Y., Stanco, A., Stumpo, D.J., Blackshear, P.J. and Anton, E.S. (2009) MARCKS Modulates Radial Progenitor Placement, Proliferation and Organization in the Developing Cerebral Cortex. Development, 136, 2965-2975. http://dx.doi.org/10.1242/dev.036616

[15] Manenti, S., Malecaze, F., Chap, H. and Darbon, J.M. (1998) Overexpression of the Myristoylated Alanine-Rich C Kinase Substrate in Human Choroidal Melanoma Cells Affects Cell Proliferation. Cancer Research, 58, 1429-1434.

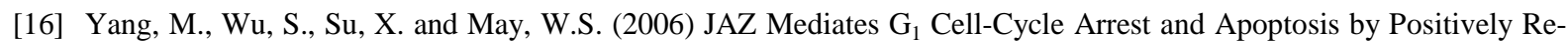
gulating p53 Transcriptional Activity. Blood, 108, 4136-4145. http://dx.doi.org/10.1182/blood-2006-06-029645

[17] Conzen, S.D., Gottlob, K., Kandel, E.S., Khanduri, P., Wagner, A.J., O’Leary, M. and Hay, N. (2000) Induction of Cell 
Cycle Progression and Acceleration of Apoptosis Are Two Separable Functions of c-Myc: Transrepression Correlates with Acceleration of Apoptosis. Molecular and Cellular Biology, 20, 6008-6018. http://dx.doi.org/10.1128/MCB.20.16.6008-6018.2000

[18] Padmanabhan, J., Park, D.S., Greene, L.A. and Shelanski, M.L. (1999) Role of Cell Cycle Regulatory Proteins in Cerebellar Granule Neuron Apoptosis. The Journal of Neuroscience, 19, 8747-8756.

[19] Monahan, T.S., Andersen, N.D., Martin, M.C., Malek, J.Y., Shrikhande, G.V., Pradhan, L., Ferran, C. and LoGerfo, F.W. (2009) MARCKS Silencing Differentially Affects Human Vascular Smooth Muscle and Endothelial Cell Phenotypes to Inhibit Neointimal Hyperplasia in Saphenous Vein. The FASEB Journal, 23, 557-564. http://dx.doi.org/10.1096/fj.08-114173

[20] McGill, C.J. and Brooks, G. (1997) Expression and Regulation of 80K/MARCKS, a Major Substrate of Protein Kinase C, in the Developing Rat Heart. Cardiovascular Research, 34, 368-376. http://dx.doi.org/10.1016/S0008-6363(97)00041-2

[21] Laouar, A., Glesne, D. and Huberman, E. (1999) Involvement of Protein Kinase C- $\beta$ and Ceramide in Tumor Necrosis Factor- $\alpha$-Induced but Not Fas-Induced Apoptosis of Human Myeloid Leukemia Cells. The Journal of Biological Chemistry, 274, 23526-23534. http://dx.doi.org/10.1074/jbc.274.33.23526

[22] Chang, Q. and Tepperman, B.L. (2001) The Role of Protein Kinase C Isozymes in TNF-Alpha-Induced Cytotoxicity to a Rat Intestinal Epithelial Cell Line. American Journal of Physiology-Gastrointestinal and Liver Physiology, 280, G572-G583.

[23] Comalada, M., Xaus, J., Valledor, A.F., López-López, C., Pennington, D.J. and Celada, A. (2003) PKC Epsilon Is Involved in JNK Activation That Mediates LPS-Induced TNF-Alpha, Which Induces Apoptosis in Macrophages. American Journal of Physiology_Cell Physiology, 285, C1235-C1245.

[24] Mong, P.Y., Petrulio, C., Kaufman, H.L. and Wang, Q. (2008) Activation of Rho Kinase by TNF-Alpha Is Required for JNK Activation in Human Pulmonary Microvascular Endothelial Cells. The Journal of Immunology, 180, 550-558. http://dx.doi.org/10.4049/jimmunol.180.1.550

[25] Ho, A.W., Wong, C.K. and Lam, C.W. (2008) Tumor Necrosis Factor-Alpha Up-Regulates the Expression of CCL2 and Adhesion Molecules of Human Proximal Tubular Epithelial Cells through MAPK Signaling Pathways. Immunobiology, 213, 533-544. http://dx.doi.org/10.1016/j.imbio.2008.01.003

[26] Okuma-Yoshioka, C., Seto, H., Kadono, Y., Hikita, A., Oshima, Y., Kurosawa, H., Nakamura, K. and Tanaka, S. (2008) Tumor Necrosis Factor-Alpha Inhibits Chondrogenic Differentiation of Synovial Fibroblasts through p38 Mitogen Activating Protein Kinase Pathways. Modern Rheumatology, 18, 366-378. http://dx.doi.org/10.3109/s10165-008-0069-5

[27] Lin, C.C., Tseng, H.W., Hsieh, H.L., Lee, C.W., Wu, C.Y., Cheng, C.Y. and Yang, C.M. (2008) Tumor Necrosis Factor-Alpha Induces MMP-9 Expression via p42/p44 MAPK, JNK, and Nuclear Factor-kappaB in A549 Cells. Toxicology and Applied Pharmacology, 15, 386-398. http://dx.doi.org/10.1016/j.taap.2008.01.032

[28] O’Sullivan, A.W., Wang, J.H. and Redmond, H.P. (2009) The Role of P38 MAPK and PKC in BLP Induced TNFAlpha Release, Apoptosis, and NFkappaB Activation in THP-1 Monocyte Cells. Journal of Surgical Research, 151, 138-144. http://dx.doi.org/10.1016/j.jss.2008.02.031

[29] Iyer, C., Kosters, A., Sethi, G., Kunnumakkara, A.B., Aggarwal, B.B. and Versalovic, J (2008) Probiotic Lactobacillus Reuteri Promotes TNF-Induced Apoptosis in Human Myeloid Leukemia-Derived Cells by Modulation of NF-kappaB and MAPK Signalling. Cellular Microbiology, 10, 1442-1452. http://dx.doi.org/10.1111/j.1462-5822.2008.01137.x

[30] Ohmitsu, M., Fukunaga, K., Yamamoto, H. and Miyamoto, E. (1999) Phosphorylation of Myristoylated Alanine-Rich Protein Kinase C Substrate by Mitogen-Activated Protein Kinase in Cultured Rat Hippocampal Neurons Following Stimulation of Glutamate Receptors. The Journal of Biological Chemistry, 274, 408-417. http://dx.doi.org/10.1074/jbc.274.1.408

[31] Nwariaku, F.E., Rothenbach, P., Liu, Z., Zhu, X., Turnage, R.H. and Terada, L.S. (2003) Rho Inhibition Decreases TNF-Induced Endothelial MAPK Activation and Monolayer Permeability. Journal of Applied Physiology, 95, 18891895. http://dx.doi.org/10.1152/japplphysiol.00225.2003

[32] Disatnik, M.H., Boutet, S.C., Lee, C.H., Mochly-Rosen, D. and Rando, T.A. (2002) Sequential Activation of Individual PKC Isozymes in Integrin-Mediated Muscle Cell Spreading: A Role for MARCKS in an Integrin Signaling Pathway. Journal of Cell Science, 115, 2151-2163.

[33] Fukushima, K., Miyamoto, S., Komatsu, H., Tsukimori, K., Kobayashi, H., Seki, H., Takeda, S. and Nakano, H. (2003) $\mathrm{TNF} \alpha$ Induced Apoptosis and Integrin Switching in Human Extravillous Trophoblast Cell Line. Biology of Reproduction, 68, 1771-1778. http://dx.doi.org/10.1095/biolreprod.102.010314

[34] Thelen, M., Rosen, A., Nairn, A.C. and Aderem, A. (1990) Tumor Necrosis Factor Alpha Modifies Agonist-Dependent Responses in Human Neutrophils by Inducing the Synthesis and Myristoylation of a Specific Protein Kinase C Sub- 
strate. Proceedings of the National Academy of Sciences of the United States of America, 87, 5603-5607. http://dx.doi.org/10.1073/pnas.87.15.5603

[35] Stein, J., Volk, H.D., Liebenthal, C.L., Krüger, D.H. and Prösch, S. (1993) Tumour Necrosis Factor $\alpha$ Stimulates the Activity of the Human Cytomegalovirus Major Immediate Early Enhancer/Promoter in Immature Monocytic Cells. Journal of General Virology, 74, 2333-2338. http://dx.doi.org/10.1099/0022-1317-74-11-2333

[36] Mandal, D., Baudin-Creuza, V., Bhattacharyya, A., Pathak, S., Delaunay, J., Kundu, M. and Basu, J. (2003) Caspase 3-Mediated Proteolysis of the N-Terminal Cytoplasmic Domain of the Human Erythroid Anion Exchanger 1 (Band 3). The Journal of Biological Chemistry, 278, 52551-52558. http://dx.doi.org/10.1074/jbc.M306914200

[37] Talanian, R.V., Dang, L.C., Ferenz, C.R., Hackett, M.C., Mankovich, J.A., Welch, J.P., Wong, W.W. and Brady, K.D. (1996) Stability and Oligomeric Equilibria of Refolded Interleukin-1 $\beta$ Converting Enzyme. The Journal of Biological Chemistry, 271, 21853-21858. http://dx.doi.org/10.1074/jbc.271.36.21853

[38] Wilson, K.P., Black, J.A., Thomson, J.A., Kim, E.E., Griffith, J.P., Navia, M.A., Murcko, M.A., Chambers, S.P., Aldape, R.A. and Raybuck, S.A. (1994) Structure and Mechanism of Interleukin-1 Beta Converting Enzyme. Nature, 370, 270-275. http://dx.doi.org/10.1038/370270a0 